\title{
Molecular Encapsulation of Cinnamaldehyde within Cyclodextrin Inclusion Complex Electrospun Nanofibers: Fast-Dissolution, Enhanced Water Solubility, High Temperature Stability, and Antibacterial Activity of Cinnamaldehyde
}

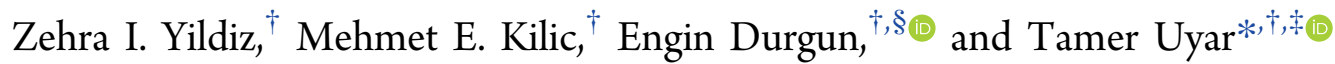 \\ ${ }^{\dagger}$ Institute of Materials Science \& Nanotechnology, Bilkent University, Ankara 06800, Turkey \\ ${ }^{\ddagger}$ Department of Fiber Science \& Apparel Design, College of Human Ecology, Cornell University, Ithaca, New York 14853, United \\ States \\ ${ }^{\S}$ UNAM-National Nanotechnology Research Center, Bilkent University, Ankara 06800, Turkey
}

Supporting Information

\begin{abstract}
The electrospinning of nanofibers (NFs) of cinnamaldehyde inclusion complexes (ICs) with two different hydroxypropylated cyclodextrins (CDs), hydroxypropyl- $\beta$-cyclodextrin (HP- $\beta$-CD) and hydroxypropyl- $\gamma$-cyclodextrin (HP- $\gamma$ CD), was successfully performed in order to produce cinnamaldehyde/CD-IC NFs without using an additional polymer matrix. The inclusion complexation between cinnamaldehyde and hydroxypropylated CDs was studied by computational molecular modeling, and the results suggested that HP- $\beta$-CD and HP- $\gamma-\mathrm{CD}$ can be inclusion complexed with cinnamaldehyde at $1: 1$ and 2:1 (cinnamaldehyde/CD) molar ratios. Additionally, molecular modeling and phase solubility studies showed that water solubility of cinnamaldehyde dramatically increases with cyclodextrin inclusion complex (CD-IC) formation. The HP- $\beta$-CD has shown slightly stronger binding with cinnamaldehyde when compared to HP- $\gamma$-CD for cinnamaldehyde/CD-IC. Although cinnamaldehyde is a highly volatile compound, it was effectively preserved with high loading by the cinnamaldehyde/CD-IC NFs. It was also observed that cinnamaldehyde has shown much higher temperature stability in cinnamaldehyde/CD-IC NFs compared to uncomplexed cinnamaldehyde because of the inclusion complexation state of cinnamaldehyde within the hydroxypropylated CD cavity. Moreover, cinnamaldehyde still has kept its antibacterial activity in cinnamaldehyde/CD-IC NF samples when tested against Escherichia coli. In addition, cinnamaldehyde/CD-IC NF mats were fast-dissolving in water, even though pure cinnamaldehyde has a water-insoluble nature. In brief, self-standing nanofibrous mats of electrospun cinnamaldehyde/CD-IC NFs are potentially applicable in food, oral-care, healthcare, and pharmaceutics because of their fast-dissolving character, enhanced water solubility, stability at elevated temperature, and promising antibacterial activity.
\end{abstract}

KEYWORDS: cinnamaldehyde, cyclodextrins, cyclodextrin inclusion complexes, electrospinning, nanofibers, fast-dissolving, water solubility, high temperature stability, antibacterial activity

\section{INTRODUCTION}

Molecular encapsulation of active agents and functional additives by cyclodextrins (CDs) has been quite practical in the food, pharmaceutical, and cosmetic industries because the $\mathrm{CD}$-encapsulated molecules can be protected from heat, light, moisture, oxygen, or other extreme conditions, thus enhancing the stability, shelf-life, and bioavailability of the active molecules. $^{1-4} \mathrm{CDs}$ which are cyclic oligosaccharides have a cone-shaped molecular structure with a hydrophobic cavity which makes them attractive host molecules for the hostguest-type inclusion complexes (ICs), especially with nonpolar compounds. ${ }^{5}$ CDs are currently used in food formulations and pharmaceutical and cosmetic products for the solubility increase, protection, and the delivery of active agents. CDs also provide controlled release in active packaging ${ }^{6}$ and they are classified as GRAS (Generally Recognized As Safe) by the U.S. Food and Drug Administration. There are mainly three classes of native CDs having six $(\alpha$-CD), seven $(\beta-\mathrm{CD})$, and eight $(\gamma$-CD) glucopyranose units linked by $\alpha-(1,4)$ linkage in their molecular structure. These three native $\mathrm{CDs}$ have relatively low water solubility; therefore, the native $\mathrm{CDs}$ are chemically modified to increase their solubility by substituting some of the primary and/or secondary hydroxyl groups by hydroxypropyl (HP) groups (Figure 1a), methyl groups, or sulfobutyl groups, and so forth.

The electrospinning (Figure $1 \mathrm{~b}$ ) technique is commonly used to produce microfibers and nanofibers (NFs) from a variety of polymeric and nonpolymeric materials. ${ }^{9-19}$ The unique properties of electrospun nanofibrous materials such as nanoporous structure, very large surface area, design flexibility, and lightweight enable their use in many applications such as food, textiles, membranes/filters, sensors, biotechnology, agriculture, composites, and energy. ${ }^{9-13}$ In general, polymeric materials are used to obtain electrospun fibrous materials because long polymeric chains provide certain chain

Received: $\quad$ May 4, 2019

Revised: July 7, 2019

Accepted: September 11, 2019

Published: September 11, 2019 
(a)
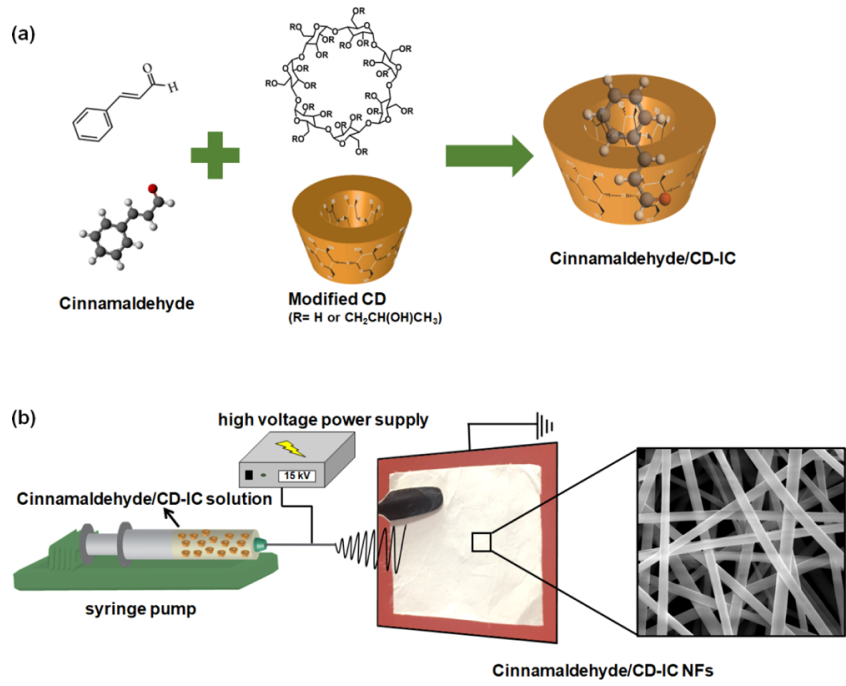

Figure 1. (a) Molecular structure of cinnamaldehyde and HP- $\beta$-CD (note: HP- $\gamma$-CD has a similar chemical structure with eight glucopyranose units), and illustration of cinnamaldehyde/CD-IC formation, (b) electrospinning process of NFs from cinnamaldehyde/ CD-IC solution.

entanglements and overlapping for the fiber formation during the electrospinning process. ${ }^{14-16}$ Hence, electrospinning of NFs purely from small molecules presents a real challenge. Nevertheless, NFs purely from CD can be electrospun, as CD molecules in their highly concentrated solutions can form substantial aggregates via hydrogen bonding. The presence of such $C D$ aggregates prevents the breakage of the jet during electrospinning, and therefore bead-free uniform NFs can be electrospun from pure CD. ${ }^{17-19}$ Furthermore, electrospun NFs from a variety of CD-ICs with food flavors and essential oils such as vanillin, ${ }^{20}$ vitamin $\mathrm{E}^{21}$ eugenol, ${ }^{22}$ menthol, ${ }^{23}$ thymol, ${ }^{24}$ and carvacrol ${ }^{25}$ were also produced successfully.

Cinnamaldehyde (Figure 1a), a primary constituent of cinnamon bark oil, is an essential oil classified as GRAS (Generally Recognized As Safe) by the U.S. Food and Drug Administration (21 CFR 182.60). Cinnamaldehyde is known to have high antibacterial, antifungal, anti-inflammatory, and antioxidant activity; therefore, it is widely used in the food, drug, and cosmetic industries. ${ }^{26-28}$ For instance, cinnamaldehyde-containing films, particles, and NFs have shown the advantages of use of cinnamaldehyde in various applications including pharmaceutics, food, and antimicrobial packaging. $^{29-33}$ For example, Qin et al. showed that active packaging including cinnamaldehyde can lead to an increase in shelf life of fresh button mushrooms. ${ }^{29}$ In another study, it was shown that submicrometer emulsion of cinnamaldehyde can be used in clinical applications because of antitumor activity and pharmacokinetic properties. $^{30}$ In the study of Liu et al., antibacterial fish skin gelatin-based NF incorporating cinnamaldehyde was fabricated to be used in controlled-release applications. ${ }^{31}$ In another study, electrospun chitosan/poly(ethylene oxide) mats including cinnamaldehyde having antibacterial activity against pseudomonas bacteria was engineered in order to be potentially used for alleviating nosocomial infections. ${ }^{32}$ In a very recent study, electrospun fibrous film was developed to be used in food industry from the pea protein isolate-polyvinyl alcohol with cinnamaldehyde cooperation to obtain a material showing antibacterial activity against both Gram-positive and -negative bacteria. ${ }^{33}$ However, cinnamaldehyde has certain shelf-life problems due to its high volatility, low stability, low solubility, and sensitivity to oxygen, light, and heat. ${ }^{34,35}$ Yet, molecular encapsulation of cinnamaldehyde with cyclodextrin inclusion complexation is one of the promising approaches in order to eliminate the abovementioned drawbacks of cinnamaldehyde. For example, it was shown that enhancement of photostability and thermostability of cinnamaldehyde after complexation with $\gamma$-CD derivatives was possible. ${ }^{36}$ In another related study, inclusion complexation between cinnamaldehyde and $\beta$-CD resulted in prevention of cinnamaldehyde from oxidation; besides, the cinnamaldehyde $/ \beta$-CD ICs showed antioxidative properties similar to pristine cinnamaldehyde. ${ }^{37}$ Moreover, the antimicrobial active packaging was designed by using ICs of cinnamaldehyde with $\beta$-CD in which improved stability and controlled release of cinnamaldehyde was obtained. ${ }^{38}$ In the study of Sun et al., the inclusion mechanism and structures of cinnamaldehyde with different cyclodextrins were investigated $^{39}$ in which HP- $\beta$-CD showed higher inclusion complexation ability with cinnamaldehyde and higher solubility enhancement for cinnamaldehyde when it was compared with inclusion complexation of $\beta$-CD. ${ }^{39}$ Moreover, several studies reported the incorporation of cyclodextrin ICs of cinnamaldehyde within the polymeric electrospun NFs for antimicrobial wound dressing and antimicrobial active food packaging. ${ }^{35,40,41}$ For instance, cinnamaldehyde/ $\beta$-CD-ICincorporated polylactic acid (PLA) nanofibrous mat was fabricated as a potential candidate for the use of wound dressing material due to its antibacterial properties and low cytotoxicity. ${ }^{35}$ Also, a polyvinyl alcohol/cinnamon essential oil/ $\beta$-CD-IC nanofibrous film was fabricated by electrospinning for active food packaging by increasing the hightemperature stability of cinnamon essential oil by inclusion complexation and prolonging the shelf life of strawberries by showing effective antimicrobial activity. ${ }^{40}$ Also, cinnamon essential oil $/ \beta$-CD-IC was incorporated into electrospun PLA NFs for antimicrobial packaging application in which the PLA/ cinnamon essential oil $/ \beta$-CD nanofibrous film has shown better antimicrobial activity compared to PLA/cinnamon essential oil nanofibrous film. ${ }^{41}$ The improvement of antimicrobial activity was attributed to cyclodextrin inclusion complexation and also, nanofibrous film obtained by the electrospinning method preserved higher amount of cinnamon essential oil when compared to film obtained by the casting method. $^{41}$

In the light of these aforementioned studies and from our extensive experiences on electrospun polymer-free CD-IC NFs, we designed our study in order to electrospin NFs from purely cinnamaldehyde/CD-IC systems without using a polymer matrix. Here, we studied inclusion complexation of cinnamaldehyde with two different hydroxypropylated CDs; hydroxypropyl- $\beta$-cyclodextrin (HP- $\beta$-CD) and hydroxypropyl- $\gamma$-cyclodextrin (HP- $\gamma-\mathrm{CD})$, in order to produce cinnamaldehyde/ CD-ICs. On the basis of our computational modeling studies, cinnamaldehyde/CD-ICs were prepared in two different molar ratios between cinnamaldehyde and CDs (cinnamaldehyde/ $\mathrm{CD} ; 1: 1$ and $2: 1$ ) in aqueous solutions. Then, aqueous solutions of cinnamaldehyde/CD-IC were electrospun in which self-standing nanofibrous mats of cinnamaldehyde/ CD-IC were produced successfully. In short, this study provides a useful approach for the molecular encapsulation of cinnamaldehyde within cyclodextrin inclusion complex NFs by electrospinning, in which fast-dissolving and highly water- 
soluble cinnamaldehyde having higher-temperature stability along with antibacterial activity was achieved for cinnamaldehyde/CD-IC NFs.

\section{MATERIALS AND METHOD}

Materials. Hydroxypropyl- $\beta$-cyclodextrin (HP- $\beta$-CD, CAVASOL W7 HP Pharma, degree of substitution: $\sim 0.6$, molecular weight: 1400 $\mathrm{g} / \mathrm{mol}$ ) and hydroxypropyl- $\gamma$-cyclodextrin (HP- $\gamma$-CD, CAVASOL W8 $\mathrm{HP}$, degree of substitution: $\sim 0.6$, molecular weight: $1574 \mathrm{~g} / \mathrm{mol}$ ) were gift samples from Wacker Chemie AG (Germany). Cinnamaldehyde (99\%, Sigma-Aldrich), 2,2-diphenyl-1-picrylhydrazyl (SigmaAldrich), deuterated dimethylsulfoxide (DMSO- $d_{6}$, deuteration degree of minimum $99.8 \%$, Merck), and deionized water (Millipore Milli-Q ultrapure water) were used in this study.

Preparation of Solutions for Electrospinning. The solutions of cinnamaldehyde/CD-IC were prepared by dissolving HP- $\beta$-CD $(200 \%, \mathrm{w} / \mathrm{v}, 2 \mathrm{~g}$ of HP- $\beta$-CD in $1 \mathrm{~mL}$ water) and HP- $\gamma$-CD $(200 \%$, $\mathrm{w} / \mathrm{v}, 2 \mathrm{~g}$ of HP- $\gamma$-CD in $1 \mathrm{~mL}$ water) in water. Then, proper amount of cinnamaldehyde was mixed with aqueous $\mathrm{CD}$ solutions in order to obtain 1:1 and 2:1 (cinnamaldehyde/CD) molar ratio of cinnamaldehyde/CD-IC solutions. These aqueous cinnamaldehyde/ CD-IC solutions were kept at room temperature under stirring for 24 $h$ in order to obtain cinnamaldehyde/HP $\beta C D-I C$ and cinnamaldehyde/HP $\gamma$ CD-IC solutions. In addition, pure CD solutions (HP- $\beta$ $\mathrm{CD}$ and $\mathrm{HP}-\gamma-\mathrm{CD})$ without cinnamaldehyde were also prepared at concentration of $200 \%(\mathrm{w} / \mathrm{v}, 2 \mathrm{~g}$ of HP- $\beta$-CD or HP- $\gamma$-CD in $1 \mathrm{~mL}$ water) in water for the electrospinning of pure CD NFs for comparative studies. ${ }^{17}$

Electrospinning of NFs. Each cinnamaldehyde/CD-IC (cinnamaldehyde/HP $\beta$ CD-IC and cinnamaldehyde/HP $\gamma \mathrm{CD}-\mathrm{IC}$ ) aqueous solution was placed separately into $1 \mathrm{~mL}$ plastic syringes having a 27 gauge metallic needle. Then, the syringe loaded with cinnamaldehyde/CD-IC aqueous solution was placed on a syringe pump (NE300, New Era Pump Systems, USA) in order to control the feed rate of the cinnamaldehyde/CD-IC solution for the electrospinning. The pumping rate of the cinnamaldehyde/CD-IC solution was $0.5 \mathrm{~mL} / \mathrm{h}$ and the electrospinning process was performed at a voltage of $\sim 15 \mathrm{kV}$ (AU Series of HV unit, Matsusada, Japan). The collection distance of the NFs was adjusted to $15 \mathrm{~cm}$, and a stationary metal plate collector covered by aluminum foil was used. In addition, pure CD NFs (HP $\beta \mathrm{CD}-\mathrm{NFs}$ and $\mathrm{HP} \gamma \mathrm{CD}-\mathrm{NFs}$ ) were also electrospun under the same condition for comparative study. ${ }^{17}$

Phase Solubility. The phase solubility test was performed and the phase solubility diagram was plotted by which stability constants of complexes between cinnamaldehyde and the two of hydroxypropylated CDs are determined. ${ }^{42}$ For the phase solubility test, $5 \mathrm{~mL}$ of the aqueous solutions of HP- $\beta$-CD and HP- $\gamma$-CD with increasing concentrations from 1 to $64 \mathrm{mM}$ were prepared in capped vials; then, cinnamaldehyde with excess amount was added to these aqueous $\mathrm{CD}$ solutions. The cinnamaldehyde/CD solutions were shaken for $48 \mathrm{~h}$ at room temperature in the dark. Then, cinnamaldehyde/CD solutions were filtered by using $0.45 \mu \mathrm{m}$ membrane filter in order to remove undissolved part if any present. After that, an UV-vis spectrophotometer (Varian, Cary 100) was used to determine the dissolved amount of cinnamaldehyde by recording the absorption at $289 \mathrm{~nm}$ in the cinnamaldehyde/CD solution. The experiment was repeated three times. The phase solubility diagram was constructed by taking the average of three measurements and plotted as cinnamaldehyde concentration versus CD concentration. The stability constants $\left(K_{\mathrm{s}}\right)$ of cinnamaldehyde/ CD-ICs were calculated as

$$
K_{\mathrm{s}}=\text { slope } / S_{0}(1-\text { slope })
$$

where $S_{0}$ is defined as the solubility of cinnamaldehyde in the absence of CD.

Computational Modeling. The ground-state properties were predicted by ab initio computational methods ${ }^{45,46}$ within the framework of density functional theory. ${ }^{43,44}$ The Perdew-BurkeErnzerhof form of generalized gradient approximation ${ }^{47}$ was used to calculate the exchange-correlation term. The weak intermolecular forces between molecules (van der Waals forces) were described by the Grimme approach. ${ }^{48}$ The projector augmented-wave method ${ }^{49}$ was implemented to describe the element potentials. The structures of HP- $\beta$-CD and HP- $\gamma$-CD were designed with 4 hydroxypropyl (HP) units (degree of substitution: $\sim 0.6$ ) and 5 HP units (degree of substitution: $\sim 0.6$ ), respectively. The structural relaxations were performed by using conjugate gradient optimization allowing $10^{-5} \mathrm{eV}$ energy tolerance between two sequential steps and allowing maximum $0.005 \mathrm{eV} / \AA$ force on atoms. The interaction between solute and solvent was analyzed by using implicit self-consistent description. ${ }^{50}$

The interaction energy $\left(E_{\text {int }}\right)$ is defined as

$$
E_{\text {int }}=E_{\mathrm{T}}(\mathrm{CD})+E_{\mathrm{T}} \text { (guest) }-E_{\mathrm{T}}(\mathrm{IC})
$$

where $E_{\mathrm{T}}(\mathrm{CD}), E_{\mathrm{T}}$ (guest), and $E_{\mathrm{T}}(\mathrm{IC})$ are the ground-state energy of HP- $\beta$-CD or HP- $\gamma$-CD, single cinnamaldehyde (for 1:1 stoichiometry) or pair of cinnamaldehyde molecules (for 2:1 stoichiometry), and their ICs, respectively. The inclusion complex is considered to be formed when $E_{\text {int }}$ is maximized and maximum value of $E_{\text {int }}$ for the given configuration is defined as complexation energy ( $\left.E_{\text {comp }}\right)$.

Solvation energy $\left(E_{\text {solv }}\right)$ is defined as

$$
E_{\text {solv }}=E_{\mathrm{T}}[\mathrm{IC}]_{\text {water }}-E_{\mathrm{T}}[\mathrm{IC}]_{\text {vacuum }}
$$

$E_{\mathrm{T}}[\mathrm{IC}]_{\text {water }}$ and $E_{\mathrm{T}}[\mathrm{IC}]_{\text {vacuum }}$ is the ground-state energy of cinnamaldehyde/HP $\beta$ CD-IC or cinnamaldehyde/HP $\gamma$ CD-IC in water and vacuum, respectively.

Measurements and Characterizations. The rheological measurement of the cinnamaldehyde/CD-IC solutions was performed via a rheometer (Anton Paar Physica MCR 301) at a $100 \mathrm{~s}^{-1}$ constant shear rate using a CP 20-4 spindle. The solution conductivities were measured by a conductivity meter (Multiparameter inoLab Multi 720WTW) at room temperature.

The morphological analyses of the samples were performed by scanning electron microscopy (SEM, FEI Quanta 200 FEG). The sputtering (Gatan 682 precision etching and coating system) was done with $5 \mathrm{~nm} \mathrm{Au} / \mathrm{Pd}$ prior to SEM imaging of nanofibrous samples. About 100 fibers from different locations of the SEM images were measured for calculating the average fiber diameter (AFD) of each nanofibrous sample.

In order to determine the presence of cinnamaldehyde in cinnamaldehyde/CD-IC electrospun nanofibrous samples, nuclear magnetic resonance spectroscopy $\left({ }^{1} \mathrm{H}\right.$ NMR, Bruker D PX-400) was used to record the ${ }^{1} \mathrm{H}$ NMR spectra. For each ${ }^{1} \mathrm{H}$ NMR spectrum, about $15 \mathrm{mg}$ of electrospun nanofibrous mat of cinnamaldehyde/CDIC NFs was dissolved in $500 \mu \mathrm{L}$ of DMSO- $d_{6}$. Fourier transform infrared (FTIR) spectra of pure cinnamaldehyde, pristine CD NFs, and cinnamaldehyde/CD-IC NFs were obtained with 64 scans at resolution of $4 \mathrm{~cm}^{-1}$ within a range of $4000-400 \mathrm{~cm}^{-1}$ using a FTIR spectrometer (Bruker VERTEX 70). For the FTIR measurements, samples were blended with potassium bromide $(\mathrm{KBr})$ and then pressed in order to obtain pellets. Thermogravimetric analyses (TGA, TA Instruments Q500) of the samples were performed from 25 to $600{ }^{\circ} \mathrm{C}$ at $20^{\circ} \mathrm{C} / \mathrm{min}$ heating rate under inert (nitrogen) atmosphere.

Antibacterial Test. The cinnamaldehyde/CD-IC NF mats were tested for their antibacterial activities against Escherichia coli. The E. coli tested was from RSHM 888 (RSHM, National Type Culture Collection Laboratory, Ankara, Turkey). E. coli was grown, and 150 $\mu \mathrm{L}$ of cultures containing approximately $10^{8} \mathrm{cfu} \mathrm{mL}^{-1}$ were spread on Luria-Bertani agar. The cinnamaldehyde/CD-IC NF mats were cut into circular pieces with $1 \mathrm{~cm}$ diameter and having the same weight. For comparison, the antibacterial activity of pristine CD-NF mats was also investigated. Each nanofibrous mat was placed separately on agar plate spread with $E$. coli and visualized after $24 \mathrm{~h}$ incubation; then, the inhibition zones (IZ) were compared.

Water Dissolution Test. Two different methods were used to visualize water dissolution of cinnamaldehyde/CD-IC NF mats. For the first method, the same weight of cinnamaldehyde/HP $\beta$ CD-IC NF and cinnamaldehyde/HP $\gamma \mathrm{CD}$-IC NF mats was positioned in a Petri dish, and then deionized water $(5 \mathrm{~mL})$ was poured directly on these 
nanofibrous mats. For the second method, the water dissolution of cinnamaldehyde/CD-IC NF mats was visualized according to the procedure modified from the literature. ${ }^{51}$ In this method, the bottom of the Petri dish was covered by absorbent paper which was wetted by $10 \mathrm{~mL}$ of distilled water. The excess water was completely drained out. Then, cinnamaldehyde/CD-IC NF mats were placed on the wet absorbent paper, and the video was recorded to visualize dissolving behavior of the samples.

\section{RESULTS AND DISCUSSION}

Phase Solubility. Figure 2 displays the phase solubility profiles of cinnamaldehyde/CD-ICs. The phase solubility

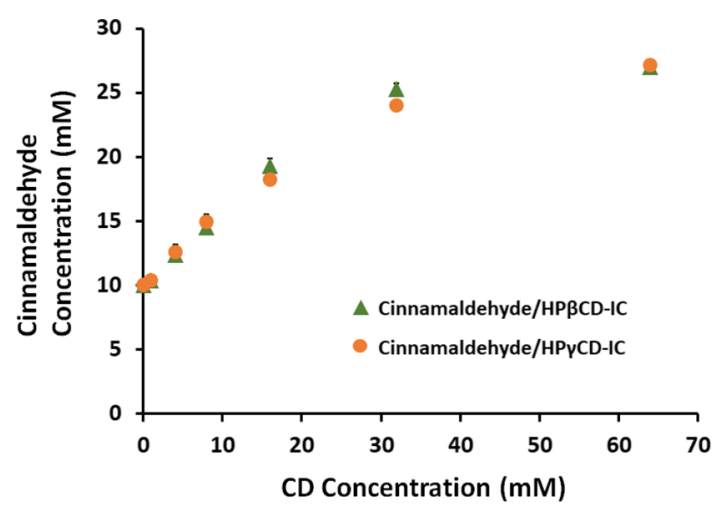

Figure 2. The phase solubility diagram of cinnamaldehyde/HP $\beta C D$ IC and cinnamaldehyde/HP $\gamma$ CD-IC in aqueous systems $(n=3)$.

diagram for cinnamaldehyde/CD-ICs shows an A type profile which indicates that solubility of cinnamaldehyde increases as a function of $C D$ concentration. ${ }^{52}$ The plots are classified as $A_{N}$ type, negative deviation from linearity which indicates that the effect of $\mathrm{CD}$ on cinnamaldehyde solubility increment was less effective at higher concentrations. For $A_{N}$ type profile, it is difficult to interpret the ratio between host and guest profiles. ${ }^{52}$ On the basis of the initial linear part of the profile, the stability constant $\left(K_{\mathrm{s}}\right.$, eq 1$)$ was calculated as 140 and $110 \mathrm{M}^{-1}\left(R^{2}>\right.$ $0.99)$ for cinnamaldehyde/HP $\beta C D-I C$ and cinnamaldehyde/ HP $\gamma$ CD-IC, respectively. The $K_{\mathrm{s}}$ values indicated that cinnamaldehyde forms stronger binding with HP- $\beta$-CD cavity when compared to HP- $\gamma$-CD cavity. The cavity of HP- $\beta-\mathrm{CD}$ is a bit smaller than HP- $\gamma$-CD, which could be a better fit and size match for cinnamaldehyde molecules, providing stronger interactions with cinnamaldehyde in cinnamaldehyde/ $\mathrm{HP} \beta \mathrm{CD}$-IC when compared to cinnamaldehyde/HP $\gamma \mathrm{CD}$-IC system. The stronger binding of cinnamaldehyde within the HP- $\beta$-CD cavity was also confirmed by molecular modeling studies.

Molecular Modeling. The molecular modeling calculations based on ab initio techniques were performed to elucidate the experimental data. Initially, HP- $\beta$-CD, HP- $\gamma$ $\mathrm{CD}$, and single cinnamaldehyde molecule (trans isomer) are relaxed in vacuum by minimizing the total energy and then optimized geometries are obtained. To reveal the complexation between the host and the guest molecules, cinnamaldehyde is moved toward HP- $\beta$-CD and HP- $\gamma$-CD through their wider rims with $1 \AA$ steps and the $E_{\text {int }}$ (eq 2) with respect to center of mass of $\mathrm{CD}$ is calculated at each step (Figure 3). This procedure is repeated for two possible orientations of cinnamaldehyde which are labeled as tail ( $-\mathrm{OH}$ group) and head (aromatic ring). As can be noticed from the Figure $3 \mathrm{a}, \mathrm{b}(\mathrm{iii}), E_{\text {int }}$ is always positive, which indicates an attractive (a)

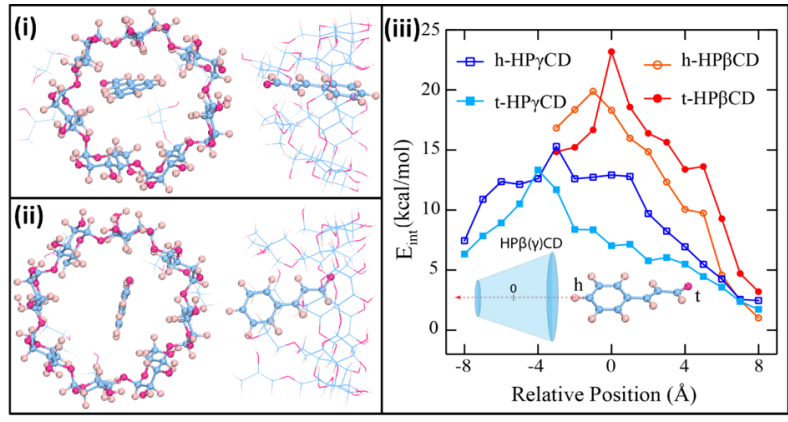

(b)

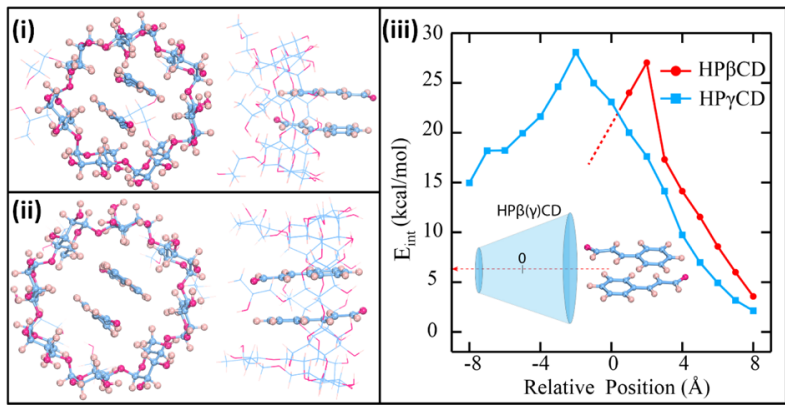

Figure 3. (a) Formation of ICs in 1:1 stoichiometry between cinnamaldehyde and CDs; (i) top and side view of cinnamaldehyde/ $\mathrm{HP} \beta \mathrm{CD}-\mathrm{IC}$, (ii) top and side view of cinnamaldehyde/HP $\gamma \mathrm{CD}-\mathrm{IC}$, and (iii) variation of interaction energy of cinnamaldehyde with $C D$ as a function of distance and (b) formation of ICs in 2:1 stoichiometry between cinnamaldehyde and CDs; (i) top and side view of cinnamaldehyde/HP $\beta$ CD-IC, (ii) top and side view of cinnamaldehyde/HP $\gamma \mathrm{CD}$-IC and (iii) variation of interaction energy of cinnamaldehyde with $\mathrm{CD}$ as a function of distance. Blue, purple, and light pink balls represent $\mathrm{C}, \mathrm{O}$, and $\mathrm{H}$ atoms, respectively.

interaction between both $\mathrm{CDs}$ and cinnamaldehyde. The variation of $E_{\text {int }}$ also demonstrates that ICs can be formed without an energy barrier and complexation is an exothermic reaction. For HP- $\beta-C D$, cinnamaldehyde perfectly fits the cavity and prefers the tail orientation with a tilt (Figure $3 a(i))$. $E_{\text {int }}$ is maximized at $0 \AA$, where cinnamaldehyde/HP $\beta$ CD-IC is assumed to be formed and $E_{\text {comp }}$ is calculated as $23.18 \mathrm{kcal}$ $\mathrm{mol}^{-1}$ (Figure 3a(iii), Table 1). For the case of HP- $\gamma-\mathrm{CD}$ (Figure 3a(ii)), cinnamaldehyde favors head orientation where the $\mathrm{OH}$-group is located in the center of $\mathrm{CD}$ and the benzene ring points toward hydroxypropyl tails. Cinnamaldehyde/ $\mathrm{HP} \gamma \mathrm{CD}$-IC is formed at $-3 \AA$ and $E_{\text {comp }}$ is calculated as 15.29 $\mathrm{kcal} \mathrm{mol}^{-1}$ (Figure 3a(iii), Table 1). When compared to HP- $\gamma$ $\mathrm{CD}$, stronger binding between HP- $\beta$ - $\mathrm{CD}$ and cinnamaldehyde is obtained for 1:1 stoichiometry. The same analysis is performed also in water to reveal the effect of the solvent. Our results indicate that although the geometries are not altered, $E_{\text {comp }}$ values significantly decrease for both cases upon interaction with water and become $18.43 \mathrm{kcal} \mathrm{mol}^{-1}$ versus $9.20 \mathrm{kcal} \mathrm{mol}^{-1}$ for cinnamaldehyde/HP $\beta \mathrm{CD}$-IC and cinnamaldehyde/HP $\gamma$ CD-IC, respectively (Table 1 ).

Next, we investigate the possibility of inclusion complex formation in the 2:1 stoichiometry (Figure 3b). The most favorable orientation for the cinnamaldehyde pair is shown in Figure $3 \mathrm{~b}$. Our results indicate that inclusion complex formation for 2:1 stoichiometry is also an exothermic process for both type of the CDs. For HP- $\beta$-CD, inclusion complex is formed at $+2 \AA$ which is partially outside the rim. HP- $\beta$-CD started to deform, if the cinnamaldehyde pair is pushed inside $($ Figure $3 \mathrm{~b}(\mathrm{i})) . E_{\text {comp }}$ slightly increases with respect to the 1:1 
Table 1. Inclusion Complexation and Solvation Energies of the Cinnamaldehyde and CDs (HP- $\beta$-CD and HP- $\gamma$-CD) at Two Different Molar Ratios (1:1 and 2:1; Cinnamaldehyde/CD) and Different Orientations

\begin{tabular}{|c|c|c|c|c|c|c|}
\hline host & guest & molar ratio (cinnamaldehyde/CD) & orientation & $E_{\text {comp }}($ vacuum $) \mathrm{kcal} \mathrm{mol}^{-1}$ & $E_{\text {comp }}($ water $) \mathrm{kcal} \mathrm{mol}^{-1}$ & $E_{\text {solv }} \mathrm{kcal} \mathrm{mol}^{-1}$ \\
\hline $\mathrm{HP}-\beta-\mathrm{CD}$ & & & & & & -71.16 \\
\hline $\mathrm{HP}-\beta-\mathrm{CD}$ & cinnamaldehyde & $1: 1$ & head & 19.88 & & \\
\hline $\mathrm{HP}-\beta-\mathrm{CD}$ & cinnamaldehyde & $1: 1$ & tail & 23.18 & 18.43 & -72.68 \\
\hline $\mathrm{HP}-\beta-\mathrm{CD}$ & cinnamaldehyde & $2: 1$ & head/tail & 27.04 & 19.79 & -75.69 \\
\hline $\mathrm{HP}-\gamma-\mathrm{CD}$ & & & & & & -83.32 \\
\hline $\mathrm{HP}-\gamma-\mathrm{CD}$ & cinnamaldehyde & $1: 1$ & head & 15.29 & 9.20 & -83.49 \\
\hline $\mathrm{HP}-\gamma-\mathrm{CD}$ & cinnamaldehyde & $1: 1$ & tail & 13.34 & & \\
\hline \multirow[t]{2}{*}{$\mathrm{HP}-\gamma-\mathrm{CD}$} & cinnamaldehyde & $2: 1$ & head/tail & 28.08 & 24.77 & -91.21 \\
\hline & cinnamaldehyde & & & & & -6.27 \\
\hline
\end{tabular}

case and becomes $27.04 \mathrm{kcal} \mathrm{mol}^{-1}$ (Figure $3 \mathrm{~b}(\mathrm{iii})$, Table 1). As the cavity of $\mathrm{HP}-\gamma-\mathrm{CD}$ is larger than that of $\mathrm{HP}-\beta-\mathrm{CD}$, the cinnamaldehyde pair perfectly fits and inclusion complex is formed at $-2 \AA$ (Figure $3 \mathrm{~b}(\mathrm{ii}))$. $E_{\text {comp }}$ drastically increases (almost doubled) and becomes $28.08 \mathrm{kcal} \mathrm{mol}^{-1}$ (Figure 3b(iii), Table 1). Similar to 1:1 stoichiometry, when calculations are repeated in water, no significant effect on geometries is noticed but $E_{\text {comp }}$ decreases for both cases and becomes 19.79 and $24.77 \mathrm{kcal} \mathrm{mol}^{-1}$ for cinnamaldehyde/ $\mathrm{HP} \beta \mathrm{CD}-\mathrm{IC}$ and cinnamaldehyde/HP $\gamma \mathrm{CD}-\mathrm{IC}$, respectively (Table 1).

Finally, solvation energies $\left(E_{\text {solv }}\right)$ are calculated by using eq 3 to reveal the solubility trends (Table 1 ). The obtained results show that although the cinnamaldehyde molecule is almost insoluble in water $\left(-6.27 \mathrm{kcal} \mathrm{mol}^{-1}\right)$, inclusion complex formation significantly increases the $E_{\text {solv }}$. These results suggest a substantial increase in cinnamaldehyde solubility. $E_{\text {solv }}$ is calculated as -72.68 and $-83.49 \mathrm{kcal} \mathrm{mol}^{-1}$ for cinnamaldehyde/HP $\beta \mathrm{CD}-\mathrm{IC}$ and cinnamaldehyde/HP $\gamma \mathrm{CD}-\mathrm{IC}$, respectively in the 1:1 stoichiometry. $E_{\text {solv }}$ further increases in the $2: 1$ stoichiometry and becomes -75.69 and $-91.21 \mathrm{kcal} \mathrm{mol}^{-1}$ for cinnamaldehyde/HP $\beta$ CD-IC and cinnamaldehyde/HP $\gamma$ CDIC, respectively.

Morphological Analyses. Optimization for the parameters of the electrospinning process was done to produce uniform NFs from cinnamaldehyde/CD-IC systems. Figure 4 displays the digital photographs of cinnamaldehyde/CD-IC mats along with their SEM images. The cinnamaldehyde/CDIC NFs without any beaded morphology were successfully electrospun, and the nanofibrous mats of cinnamaldehyde/ CD-IC NFs were obtained having self-standing, easy-to-handle, lightweight, and flexible features. Table 2 summarizes the conductivity and viscosity of the highly concentrated cinnamaldehyde/HP $\beta$ CD-IC and cinnamaldehyde/HP $\gamma$ CDIC aqueous solutions and the AFD of the resulting electrospun cinnamaldehyde/HP $\beta$ CD-IC NFs and cinnamaldehyde/ $\mathrm{HP} \gamma \mathrm{CD}-\mathrm{IC}$ NFs for 1:1 and 2:1 molar ratios. It is a known fact that the conductivity and viscosity of the solutions noticeably affect the electrospinnability and resulting diameter of the electrospun NFs. Typically, less stretching occurs during the electrospinning process when the solution has higher viscosity and lower conductivity, and therefore, much thicker fibers are electrospun. ${ }^{53}$ When compared to other cinnamaldehyde/CD-IC NFs, cinnamaldehyde/HP $\gamma$ CD-IC NFs (1:1) have the highest value of AFD which was measured as $825 \pm$ $330 \mathrm{~nm}$, as its solution has the highest viscosity $(2.18 \pm 0.65$ $\mathrm{Pa} \mathrm{s})$ and the lowest conductivity $\left(4.53 \pm 0.30 \mu \mathrm{S} \mathrm{cm} \mathrm{cm}^{-1}\right)$ among other cinnamaldehyde/CD-IC solutions. The cinnamaldehyde/HP $\gamma$ CD-IC NFs (2:1) has AFD value of $805 \pm$
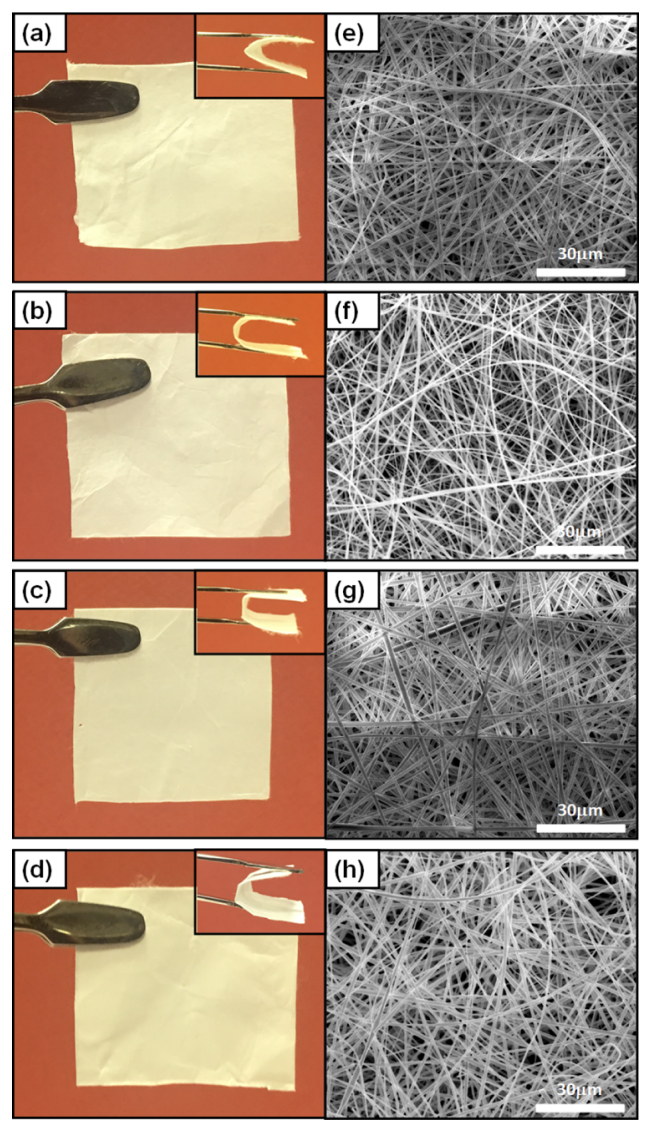

Figure 4. The digital photograph of (a) cinnamaldehyde/HP $\beta$ CD-IC NF mat (1:1), (b) cinnamaldehyde/HP $\beta$ CD-IC NF mat (2:1), (c) cinnamaldehyde/HP $\gamma \mathrm{CD}-\mathrm{IC} \mathrm{NF}$ mat $(1: 1)$, and (d) cinnamaldehyde/HP $\gamma$ CD-IC NFs $(2: 1)$ mat. The representative SEM images of (e) cinnamaldehyde/HP $\beta$ CD-IC NFs (1:1), (f) cinnamaldehyde/ HP $\beta$ CD-IC NFs (2:1), (g) cinnamaldehyde/HP $\gamma$ CD-IC NFs $(1: 1)$, and $(\mathrm{h})$ cinnamaldehyde/HP $\gamma \mathrm{CD}-\mathrm{IC}$ NFs $(2: 1)$.

$275 \mathrm{~nm}$ which is slightly higher than AFD of NFs obtained from cinnamaldehyde/HP $\beta$ CD-ICs in both 1:1 (700 \pm 260 $\mathrm{nm})$ and 2:1 $(545 \pm 175 \mathrm{~nm})$ molar ratios. The reason for this difference in AFD is that solution of cinnamaldehyde/HP $\gamma \mathrm{CD}$ IC $(2: 1)$ has higher viscosity $(1.84 \pm 0.30 \mathrm{~Pa} \mathrm{~s})$ and lower conductivity $\left(5.00 \pm 0.16 \mu \mathrm{S} \mathrm{cm} \mathrm{cm}^{-1}\right)$ than solutions of cinnamaldehyde/HP $\beta$ CD-IC. In contrast, cinnamaldehyde/ $\mathrm{HP} \beta \mathrm{CD}-\mathrm{IC}$ NFs $(2: 1)$ has the lowest AFD value $(545 \pm 175$ $\mathrm{nm})$ due to its lowest viscosity which is $1.15 \pm 0.16 \mathrm{~Pa}$ s and higher conductivity which is $12.18 \pm 0.25 \mu \mathrm{S} \mathrm{cm}^{-1}$. Overall, the trends in AFD values of cinnamaldehyde/CD-IC NFs were well correlated with the electrospun polymeric NFs, where 
Table 2. Solutions Properties (Viscosity and Conductivity) and the Diameter of the Resulting Electrospun Cinnamaldehyde/ CD-IC NFs

\begin{tabular}{lccc} 
samples & viscosity $(\mathrm{Pa} \mathrm{s})$ & conductivity $\left(\mu \mathrm{S} \mathrm{cm}^{-1}\right)$ & average fiber diameter $(\mathrm{nm})$ \\
\hline cinnamaldehyde/HP $\beta$ CD-IC (1:1) & $1.76 \pm 0.29$ & $12.66 \pm 0.65$ & $700 \pm 260$ \\
cinnamaldehyde/HP $\beta$ CD-IC (2:1) & $1.15 \pm 0.16$ & $12.18 \pm 0.25$ & $545 \pm 175$ \\
cinnamaldehyde/HP $\gamma$ CD-IC (1:1) & $2.18 \pm 0.65$ & $4.53 \pm 0.30$ & $825 \pm 330$ \\
cinnamaldehyde/HP $\gamma$ CD-IC (2:1) & $1.84 \pm 0.30$ & $5.00 \pm 0.16$ & $805 \pm 275$
\end{tabular}

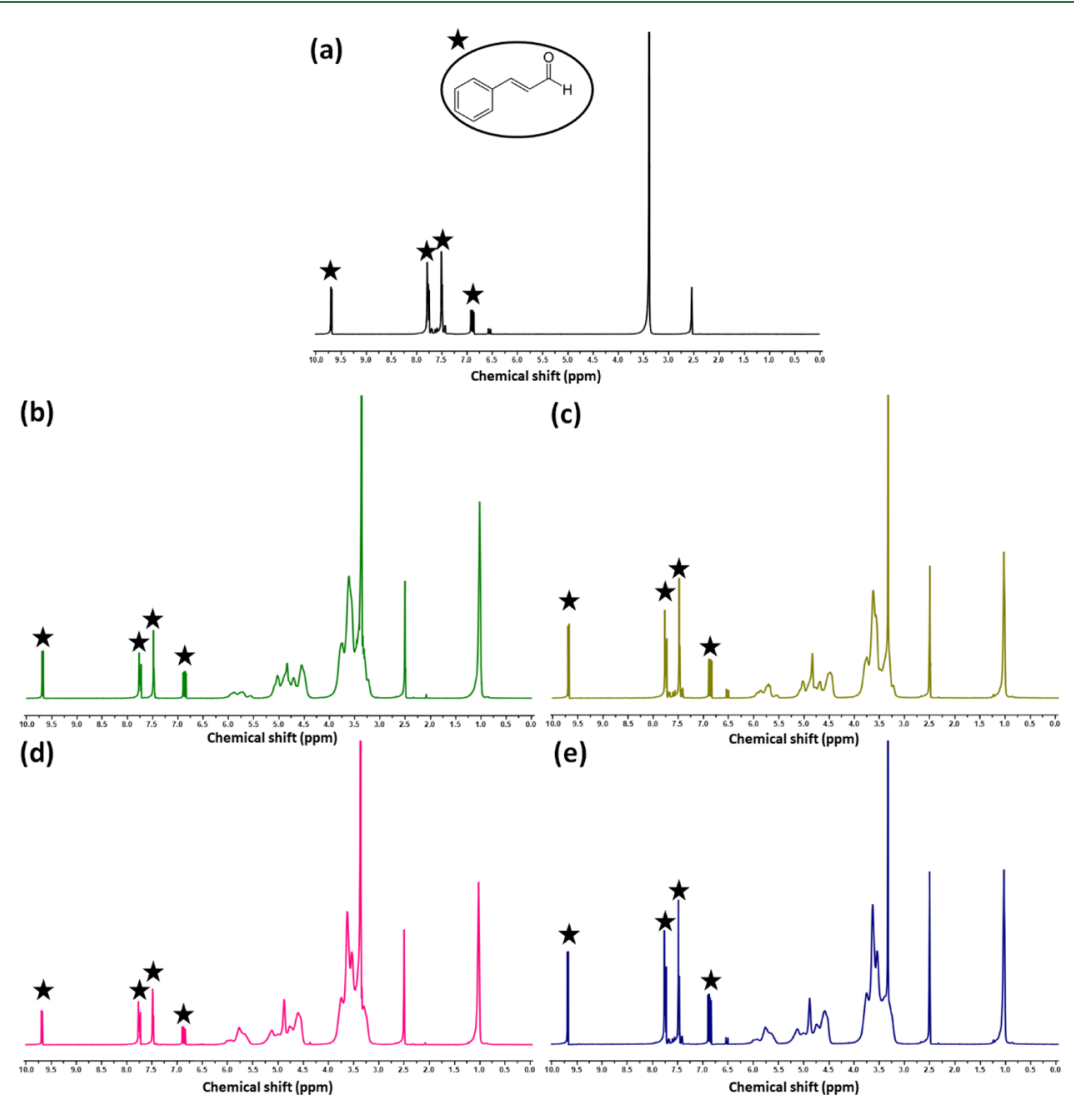

Figure 5. The ${ }^{1} \mathrm{H}$ NMR spectra of (a) pure cinnamaldehyde, (b) cinnamaldehyde/HP $\beta$ CD-IC NFs (1:1), (c) cinnamaldehyde/HP $\beta$ CD-IC NFs (2:1), (d) cinnamaldehyde/HP $\gamma$ CD-IC NFs (1:1), and (e) cinnamaldehyde/HP $\gamma$ CD-IC NFs $(2: 1)$. The ${ }^{1} \mathrm{H}$ NMR spectra were recorded by dissolving the samples in DMSO- $d_{6}$. The characteristic peaks of cinnamaldehyde are shown by black stars.

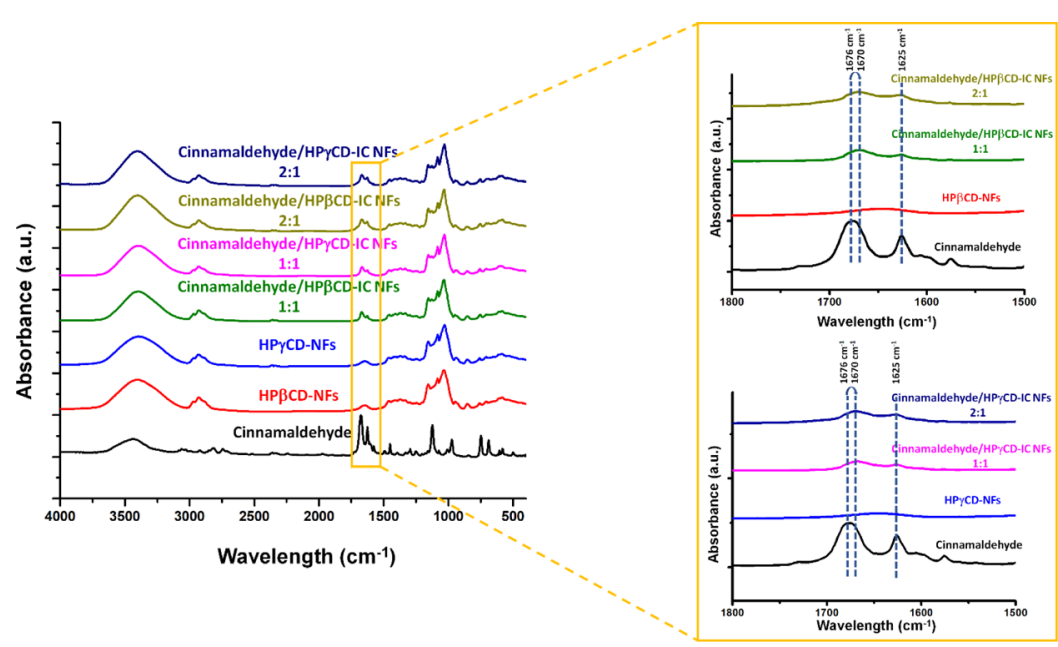

Figure 6. The FTIR spectra of pure cinnamaldehyde, pristine CD NFs, and cinnamaldehyde/CD-IC NFs.

lower conductivity and higher viscosity resulted in much bigger fibers. 53
Structural Characterization. The presence of cinnamaldehyde in electrospun cinnamaldehyde/HP $\beta$ CD-IC NFs was 


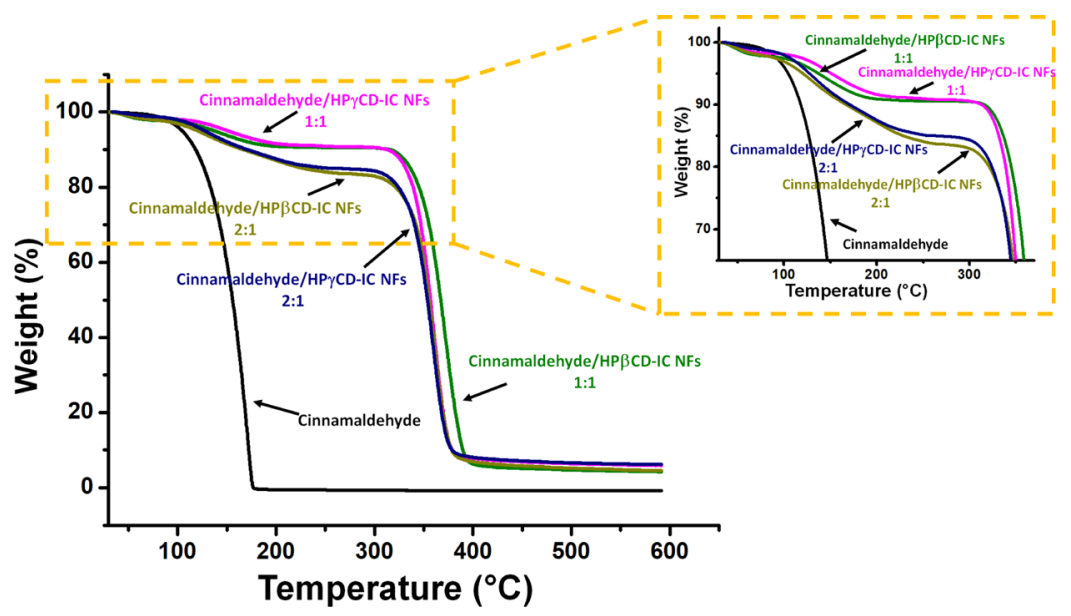

Figure 7. The TGA thermogram of pure cinnamaldehyde and cinnamaldehyde/CD-IC NFs.

validated by ${ }^{1} \mathrm{H}$ NMR study (Figure 5 ). The specific peaks of cinnamaldehyde were detected in ${ }^{1} \mathrm{H}$ NMR spectra of all cinnamaldehyde/CD-IC NFs samples (Figure 5) confirming the presence of cinnamaldehyde in these samples. Although cinnamaldehyde is highly volatile, the ${ }^{1} \mathrm{H}$ NMR study clearly elucidated that cinnamaldehyde was effectively preserved in all cinnamaldehyde/CD-IC NFs samples during the whole process, including preparation of CD-IC solutions as well as during the electrospinning process of cinnamaldehyde/CD-IC NFs and their storage.

FTIR spectroscopy is a useful technique to study the cyclodextrin inclusion complexation between host and guest molecules. ${ }^{54}$ Moreover, FTIR spectroscopy can also verify the presence of guest molecules in the CD-IC samples. In FTIR analysis, inclusion complex formation can cause variations for the peak position and peak intensity for the guest molecules when they are in the complexation state with the host CD cavity. The FTIR spectrum of cinnamaldehyde, pristine CD NFs, and cinnamaldehyde/CD-IC NFs are shown in Figure 6. The characteristic peaks for cinnamaldehyde at $1625 \mathrm{~cm}^{-1}$ belonging to the $\mathrm{C}=\mathrm{C}$ double bond and $1676 \mathrm{~cm}^{-1}$ belonging to the $\mathrm{C}=\mathrm{O}$ double bond were detected clearly. In the case of ICs, these peaks were present and the intensity of these peaks were suppressed; besides, the peak at $1676 \mathrm{~cm}^{-1}$ for the $\mathrm{C}=\mathrm{C}$ double bond was shifted to $1670 \mathrm{~cm}^{-1}$, whereas they were not observed at the spectra of pristine CD NFs. This type of peak position shift and peak intensity suppression is typically observed for CD-IC systems. Therefore, FTIR results suggested that the guest cinnamaldehyde molecules are in the complexation state with the host CDs (HP- $\beta$-CD and HP$\gamma$-CD, $1: 1$ and 2:1 molar ratio) in all cinnamaldehyde/CD-IC NFs samples.

Thermal Analyses. The TGA data of pure cinnamaldehyde, cinnamaldehyde/HP $\beta$ CD-IC NFs (1:1 and 2:1 molar ratio), and cinnamaldehyde/HP $\gamma \mathrm{CD}-\mathrm{IC}$ NFs (1:1 and 2:1 molar ratio) are given in Figure 7. As cinnamaldehyde is a volatile organic compound, the evaporation of pure cinnamaldehyde starts at a low temperature (below $50{ }^{\circ} \mathrm{C}$ ) and it completely vaporized before the temperature of $200{ }^{\circ} \mathrm{C}$. For cinnamaldehyde/CD-IC NFs samples, there are three weight losses observed; the weight loss below $\sim 100{ }^{\circ} \mathrm{C}$ is due to water loss, and the weight loss above $300{ }^{\circ} \mathrm{C}$ is due to main thermal decomposition of hydroxypropylated $\mathrm{CD}$ molecules. The weight loss step observed between 100 and $300{ }^{\circ} \mathrm{C}$ is due to vaporization of cinnamaldehyde in cinnamaldehyde/CD-IC NFs. For cinnamaldehyde/HP $\beta$ CD-IC NFs (1:1), the evaporation of cinnamaldehyde started at $\sim 85{ }^{\circ} \mathrm{C}$ and completed at $\sim 240{ }^{\circ} \mathrm{C}$, whereas for cinnamaldehyde/ HP $\beta$ CD-IC NFs (2:1), the evaporation of cinnamaldehyde was observed between $\sim 75$ and $270{ }^{\circ} \mathrm{C}$. On the other hand, for cinnamaldehyde/HP $\gamma \mathrm{CD}-\mathrm{IC}$ NFs (1:1), the evaporation of cinnamaldehyde was observed between $\sim 85$ and $\sim 260{ }^{\circ} \mathrm{C}$, whereas the evaporation of cinnamaldehyde was observed between $\sim 75$ and $\sim 270{ }^{\circ} \mathrm{C}$ for cinnamaldehyde/ $\mathrm{HP} \gamma \mathrm{CD}$-IC NFs (2:1). The TGA studies clearly showed that the evaporation of cinnamaldehyde takes place at a much higher temperature for cinnamaldehyde/CD-IC NFs samples compared to pure cinnamaldehyde. The higher evaporation temperature of cinnamaldehyde in cinnamaldehyde/CD-IC NF mats elucidates the inclusion complexation between guest cinnamaldehyde and host CD molecules. Also, the presence of guest-host inclusion complexation in these cinnamaldehyde/ CD-IC NF samples provides much higher thermal stability for the cinnamaldehyde.

The weight percentage of cinnamaldehyde present in cinnamaldehyde/CD-IC NF mats was calculated from TGA thermograms. The amount of cinnamaldehyde was calculated as $7.30 \%(\mathrm{w} / \mathrm{w}), 7.20 \%(\mathrm{w} / \mathrm{w}), 14.30 \%(\mathrm{w} / \mathrm{w})$, and $13.70 \%$ $(\mathrm{w} / \mathrm{w})$ for cinnamaldehyde/HP $\beta$ CD-IC NFs (1:1), cinnamaldehyde/HP $\gamma$ CD-IC NFs (1:1), cinnamaldehyde/HP $\beta$ CD-IC NFs (2:1), and cinnamaldehyde/HP $\gamma$ CD-IC NFs (2:1), respectively. The initial theoretical amount of cinnamaldehyde is $8.25 \%(\mathrm{w} / \mathrm{w})$ in cinnamaldehyde/HP $\beta$ CD-IC NFs (1:1), $7.32 \%(\mathrm{w} / \mathrm{w})$ in cinnamaldehyde/HP $\gamma \mathrm{CD}-\mathrm{IC}$ NFs (1:1), $15.25 \%(\mathrm{w} / \mathrm{w})$ in cinnamaldehyde/HP $\beta$ CD-IC NFs (2:1), and $13.79 \%(\mathrm{w} / \mathrm{w})$ in cinnamaldehyde/HP $\gamma$ CD-IC NFs $(2: 1)$, respectively. Therefore, it was concluded that cinnamaldehyde was mostly preserved with a very high efficiency $(\sim 90 \%, \mathrm{w} / \mathrm{w})$ within the cinnamaldehyde/CD-IC NFs, although cinnamaldehyde is a highly volatile organic compound.

Antibacterial Activity. The antibacterial activities of cinnamaldehyde/HP $\beta$ CD-IC NFs and cinnamaldehyde/ $\mathrm{HP} \gamma \mathrm{CD}-\mathrm{IC}$ NFs for both molar ratios (1:1 and 2:1 molar ratio) were investigated against strains of $E$. coli by the agar diffusion method. E. coli is a Gram-negative bacteria which is one of the significant food-borne pathogens that can cause serious disease outbreaks all over the world. ${ }^{55}$ There is an increasing demand on the use of natural sterilization to prevent 
possible infections caused by E. coli or some other undesirable food-related microorganisms instead of the use of chemical food preservatives. ${ }^{55,56}$ The use of essential oils derived from plants have received great attention as a natural source to inhibit food-borne pathogens. ${ }^{55-58}$ Among plant essential oils, cinnamaldehyde was reported as one of the effective essential oils against E. coli. ${ }^{58-60}$ The pristine CDs do not show antibacterial activity against E. coli, and so the NFs purely based on CDs are not expected to show any antibacterial activity. ${ }^{61}$ Nevertheless, in our study, the antibacterial activity test against $E$. coli for pristine $\mathrm{CD}$ NFs was also done as a control test in order to see the differences caused by the presence of cinnamaldehyde in cinnamaldehyde/CD-IC NF mats (Figure 8). As expected, pristine CD NFs did not show
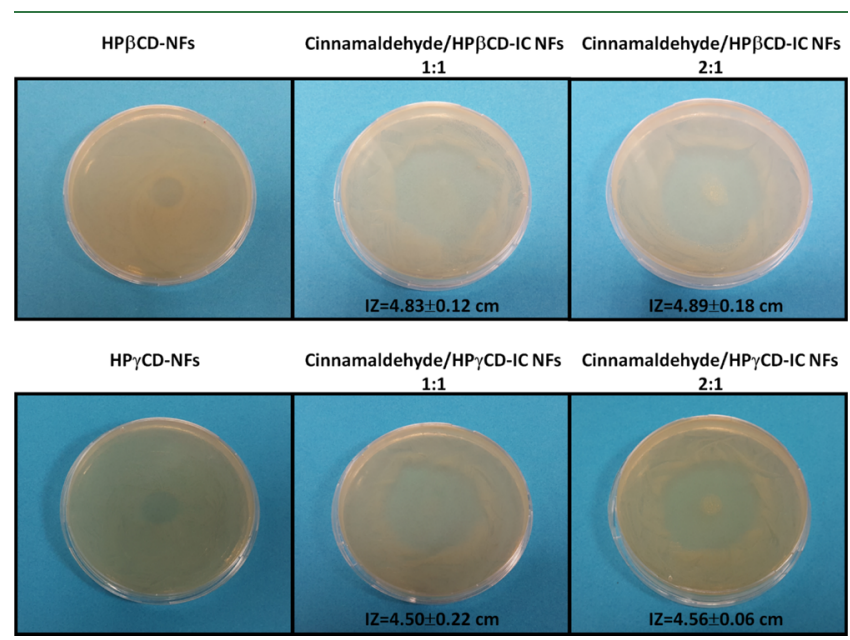

Figure 8. Representative digital photographs of antibacterial test plates of pristine CD NF mats and cinnamaldehyde/CD-IC NF mats against $E$. coli. The average IZ obtained from agar diffusion method ( $n$ $=3)$.

any inhibition against $E$. coli growth. When the antibacterial activity against $E$. coli was tested for cinnamaldehyde/CD-IC NFs samples, the diameter of the IZs was observed as $4.83 \pm$ $0.12,4.89 \pm 0.18,4.50 \pm 0.22$, and $4.56 \pm 0.06 \mathrm{~cm}$ for cinnamaldehyde/HP $\beta$ CD-IC NFs (1:1), cinnamaldehyde/ HP $\beta$ CD-IC NFs (2:1), cinnamaldehyde/HP $\gamma$ CD-IC NFs (1:1), and cinnamaldehyde/HP $\gamma \mathrm{CD}-\mathrm{IC}$ NFs (2:1), respectively. These results indicated that cinnamaldehyde/CD-IC NFs has certain amount of cinnamaldehyde which still effectively shows antibacterial activity against $E$. coli.

Water Dissolution Test. The water solubility of cinnamaldehyde is quite low $\left(1.35 \mathrm{~g} \mathrm{~L}^{-1}\right){ }^{62}$ which sometimes limits its applications. However, as revealed from phase solubility and molecular modeling studies, CD-IC formation significantly enhanced the water solubility of cinnamaldehyde. Here, two different methods were used to visualize the water solubility enhancement and fast-dissolution behavior of cinnamaldehyde/CD-IC NF mats (Videos S1 and S2). For the first method, distilled water was directly added to the cinnamaldehyde/CD-IC NF mats. As seen from Figure 9 and Video S1, both cinnamaldehyde/HP $\beta$ CD-IC NFs (1:1 and 2:1 molar ratio) and cinnamaldehyde/HP $\gamma$ CD-IC NFs (1:1 and 2:1 molar ratio) samples show very fast-dissolution behavior with the addition of water. Because of the nanofibrous structure providing very high surface area, cinnamaldehyde/ CD-IC NF mats were dissolved in water very quickly (less than

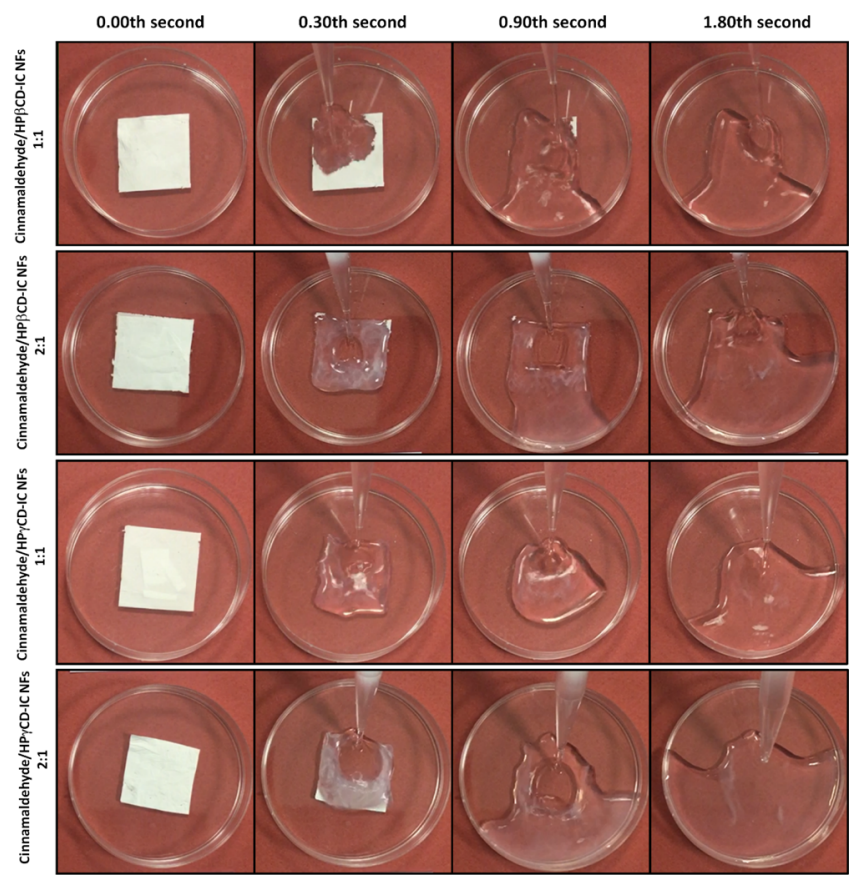

Figure 9. Representation of dissolution behavior of cinnamaldehyde/ CD-IC NF mats when exposed to distilled water (the images were captured from the Video S1). The cinnamaldehyde/CD-IC NF mats are dissolved completely in a few seconds.

a second), which proves the fast-dissolving character of cinnamaldehyde/CD-IC NF mats. For the second method, absorbent paper was soaked by distilled water, then cinnamaldehyde/CD-IC NF mats were placed onto this paper. Figure 10 and Video S2 shows the dissolution character of cinnamaldehyde/CD-IC NF mats. The cinnamaldehyde/ CD-IC NF mats were dissolved instantly when they were placed onto the distilled water-soaked absorbent paper. This result clearly shows that these cinnamaldehyde/CD-IC NF mats have ultrafast dissolution behavior even in the presence of a small amount of water such as saliva. These results are very promising that the cinnamaldehyde/CD-IC NF mats can be used in variety of applications including fast-dissolving oral care products. In both dissolution tests, it was observed that cinnamaldehyde/CD-IC NFs samples having 2:1 (cinnamaldehyde/CD) molar ratio have shown slightly slower dissolution behavior compared to cinnamaldehyde/CD-IC NFs samples having 1:1 (cinnamaldehyde/CD) molar ratio. This is possibly because of the higher content of the cinnamaldehyde present in cinnamaldehyde/CD-IC NFs samples having 2:1 molar ratio which may slightly decrease the dissolution rate of these nanofibrous mats.

In brief, cinnamaldehyde was inclusion complexed with two different type of hydroxypropylated CDs (HP- $\beta$-CD and HP- $\gamma$ $\mathrm{CD})$ at two different molar ratios (1:1 and $2: 1$, cinnamaldehyde/CD). The inclusion complexation of cinnamaldehyde with $\mathrm{HP}-\beta-\mathrm{CD}$ and $\mathrm{HP}-\gamma-\mathrm{CD}$ was investigated by phase solubility and molecular modeling studies. The electrospinning of NFs from cinnamaldehyde/CD-IC was successfully performed. Although cinnamaldehyde is a highly volatile organic compound, because of the inclusion complexation within the $C D$ cavity, cinnamaldehyde was effectively preserved with high efficiency in electrospun cinnamaldehyde/CD-IC NFs during the whole process including preparation, electrospinning, and storage. Cinnamaldehyde 


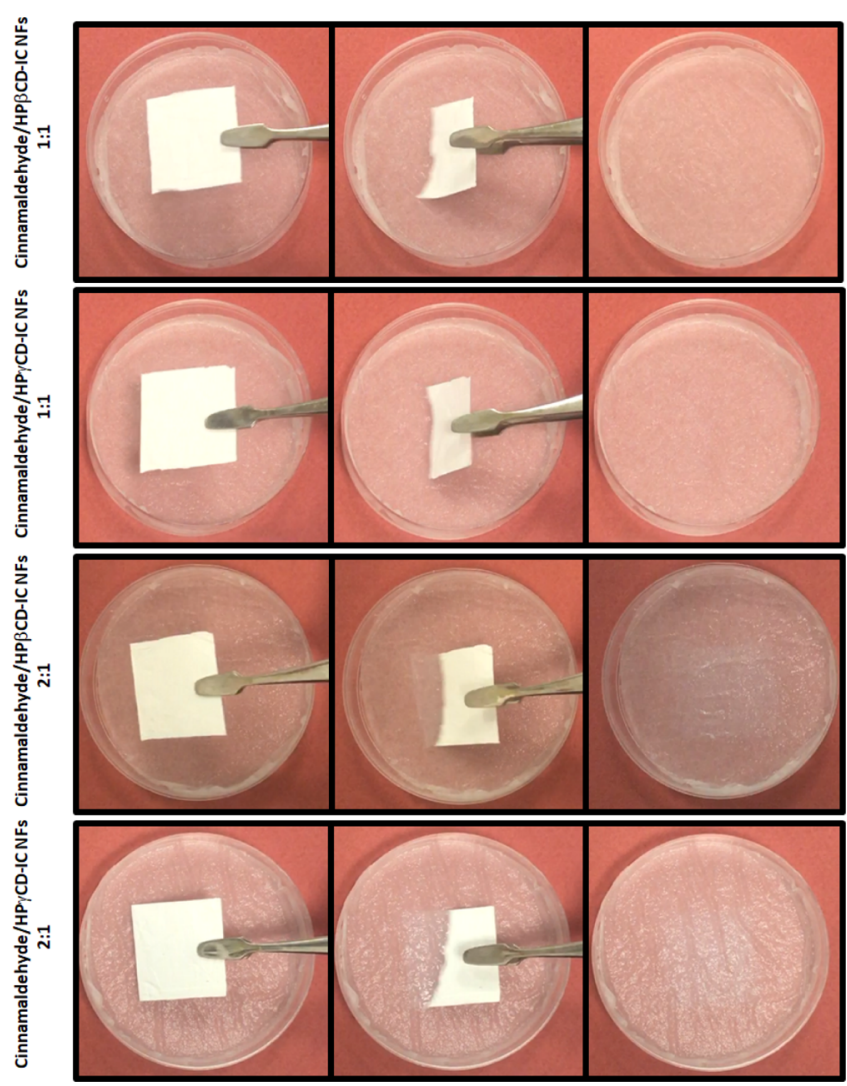

Figure 10. Representation of dissolution behavior of cinnamaldehyde/CD-IC NF mats when contacted to water soaked absorbent paper (the pictures were captured from the Video S2). The cinnamaldehyde/CD-IC NF mats are dissolved instantly.

has shown much higher temperature stability in cinnamaldehyde/CD-IC NFs when compared to its pure form due to the inclusion complexation. The cinnamaldehyde/CD-IC NFs have shown antibacterial activity when tested against E. coli. Even though cinnamaldehyde is not soluble in water, cinnamaldehyde/CD-IC NF mats have shown fast-dissolving character in water. These promising results suggest that cinnamaldehyde/CD-IC NF mats may be quite applicable in food, oral-care, healthcare, pharmaceutics, cosmetics, and so forth, due to their fast-dissolution, enhanced water solubility, high temperature stability, and promising antibacterial activity.

\section{ASSOCIATED CONTENT}

\section{S Supporting Information}

The Supporting Information is available free of charge on the ACS Publications website at DOI: 10.1021/acs.jafc.9b02789.

Dissolution behavior of cinnamaldehyde/CD-IC NF mats when exposed to distilled water (MP4)

Representation of dissolution behavior of cinnamaldehyde/CD-IC NF mats when contacted to water soaked absorbent paper (MP4)

\section{AUTHOR INFORMATION}

\section{Corresponding Author}

*E-mail: tu46@cornell.edu.

\section{ORCID $\odot$}

Engin Durgun: 0000-0002-0639-5862

Tamer Uyar: 0000-0002-3989-4481

\section{Author Contributions}

T.U. designed the study. Z.I.Y. performed the experiment studies. M.E.K. and E.D. performed the molecular modeling studies. All authors contributed to writing of the manuscript and have given approval to the final version of the manuscript.

Notes

The authors declare no competing financial interest.

\section{ACKNOWLEDGMENTS}

TUBITAK-BIDEB is acknowledged for supporting $\mathrm{PhD}$ scholarship to Z.I.Y. High Performance and Grid Computing Center (TR-Grid e-Infrastructure), TUBITAK ULAKBIM and the National Center for High Performance Computing of Turkey (UHeM) under grant no. 5003622015 are used for the computational resources.

\section{REFERENCES}

(1) Bilensoy, E. Cyclodextrins in Pharmaceutics, Cosmetics, and Biomedicine: Current and Future Industrial Applications, Wiley: Hoboken, N.J., 2011; p x, 395, 16 p. of plates.

(2) dos Santos, C.; Buera, P.; Mazzobre, F. Novel trends in cyclodextrins encapsulation. Applications in food science. Curr. Opin. Food Sci. 2017, 16, 106-113.

(3) Fenyvesi, É.; Vikmon, M.; Szente, L. Cyclodextrins in Food Technology and Human Nutrition: Benefits and Limitations. Crit. Rev. Food Sci. 2016, 56, 1981-2004.

(4) Astray, G.; Gonzalez-Barreiro, C.; Mejuto, J. C.; Rial-Otero, R.; Simal-Gandara, J. A review on the use of cyclodextrins in foods. Food Hydrocolloids 2009, 23, 1631-1640.

(5) Szejtli, J. Introduction and General Overview of Cyclodextrin Chemistry. Chem. Rev. 1998, 98, 1743-1754.

(6) Duarte, A.; Martinho, A.; Luis, A.; Figueiras, A.; Oleastro, M.; Domingues, F. C.; Silva, F. Resveratrol encapsulation with methylbeta-cyclodextrin for antibacterial and antioxidant delivery applications. LWT-Food Sci. Technol. 2015, 63, 1254-1260.

(7) Szente, L.; Szejtli, J. Highly soluble cyclodextrin derivatives: chemistry, properties, and trends in development. Adv. Drug Delivery Rev. 1999, 36, 17-28.

(8) Albers, E.; Müller, B. W. Cyclodextrin derivatives in pharmaceutics. Crit. Rev. Ther. Drug Carrier Syst. 1995, 12, 311-337.

(9) Uyar, T.; Kny, E. Electrospun Materials for Tissue Engineering and Biomedical Applications; Woodhead Publishing, 2017.

(10) Noruzi, M. Electrospun nanofibres in agriculture and the food industry: a review. J. Sci. Food Agric. 2016, 96, 4663-4678.

(11) Aytac, Z.; Keskin, N. O. S.; Tekinay, T.; Uyar, T., Antioxidant alpha-tocopherol/gamma-cyclodextrin-inclusion complex encapsulated poly(lactic acid) electrospun nanofibrous web for food packaging. J. Appl. Polym. Sci. 2017, 134, 44858.

(12) Senthamizhan, A.; Balusamy, B.; Uyar, T. Glucose sensors based on electrospun nanofibers: a review. Anal. Bioanal. Chem. 2016, 408, 1285-1306.

(13) Xue, J.; Wu, T.; Dai, Y.; Xia, Y. Electrospinning and Electrospun Nanofibers: Methods, Materials, and Applications. Chem. Rev. 2019, 119, 5298-5415.

(14) Ramakrishna, S.; Fujihara, K.; Teo, W. E.; Lim, T. C.; Ma, Z. An Introduction to Electrospinning and Nanofibers; World Scientific, 2005.

(15) Shenoy, S. L.; Bates, W. D.; Frisch, H. L.; Wnek, G. E. Role of chain entanglements on fiber formation during electrospinning of polymer solutions: good solvent, non-specific polymer-polymer interaction limit. Polymer 2005, 46, 3372-3384.

(16) Theron, S. A.; Zussman, E.; Yarin, A. L. Experimental investigation of the governing parameters in the electrospinning of polymer solutions. Polymer 2004, 45, 2017-2030.

(17) Celebioglu, A.; Uyar, T. Electrospinning of nanofibers from non-polymeric systems: polymer-free nanofibers from cyclodextrin derivatives. Nanoscale 2012, 4, 621-631. 
(18) Celebioglu, A.; Uyar, T. Electrospinning of nanofibers from non-polymeric systems: electrospun nanofibers from native cyclodextrins. J. Colloid Interface Sci. 2013, 404, 1-7.

(19) Topuz, F.; Uyar, T. Influence of Hydrogen-Bonding Additives on Electrospinning of Cyclodextrin Nanofibers. ACS Omega 2018, 3, 18311-18322.

(20) Celebioglu, A.; Kayaci-Senirmak, F.; İpek, S.; Durgun, E.; Uyar, T. Polymer-free nanofibers from vanillin/cyclodextrin inclusion complexes: high thermal stability, enhanced solubility and antioxidant property. Food Funct. 2016, 7, 3141-3153.

(21) Celebioglu, A.; Uyar, T. Antioxidant Vitamin E/Cyclodextrin Inclusion Complex Electrospun Nanofibers: Enhanced Water Solubility, Prolonged Shelf Life, and Photostability of Vitamin E. J. Agric. Food Chem. 2017, 65, 5404-5412.

(22) Celebioglu, A.; Yildiz, Z. I.; Uyar, T. Fabrication of Electrospun Eugenol/Cyclodextrin Inclusion Complex Nanofibrous Webs for Enhanced Antioxidant Property, Water Solubility, and High Temperature Stability. J. Agric. Food Chem. 2018, 66, 457-466.

(23) Yildiz, Z. I.; Celebioglu, A.; Kilic, M. E.; Durgun, E.; Uyar, T. Menthol/cyclodextrin inclusion complex nanofibers: Enhanced water solubility and high-temperature stability of menthol. J. Food Eng. 2018, 224, 27-36.

(24) Celebioglu, A.; Yildiz, Z. I.; Uyar, T. Thymol/cyclodextrin inclusion complex nanofibrous webs: Enhanced water solubility, high thermal stability and antioxidant property of thymol. Food Res. Int. 2018, 106, 280-290.

(25) Yildiz, Z. I.; Celebioglu, A.; Kilic, M. E.; Durgun, E.; Uyar, T. Fast-dissolving carvacrol/cyclodextrin inclusion complex electrospun fibers with enhanced thermal stability, water solubility, and antioxidant activity. J. Mater. Sci. 2018, 53, 15837-15849.

(26) Nabavi, S.; Di Lorenzo, A.; Izadi, M.; Sobarzo-Sánchez, E.; Daglia, M.; Nabavi, S. Antibacterial Effects of Cinnamon: From Farm to Food, Cosmetic and Pharmaceutical Industries. Nutrients 2015, 7, $7729-7748$.

(27) Chen, B.-J.; Fu, C.-S.; Li, G.-H.; Wang, X.-N.; Lou, H.-X.; Ren, D.-M.; Shen, T. Cinnamaldehyde Analogues as Potential Therapeutic Agents. Mini-Rev. Med. Chem. 2017, 17, 33-43.

(28) Gunia-Krzyzak, A.; Sloczynska, K.; Popiol, J.; Koczurkiewicz, P.; Marona, H.; Pekala, E. Cinnamic acid derivatives in cosmetics: current use and future prospects. Int. J. Cosmet. Sci. 2018, 40, 356366.

(29) Qin, Y. Y.; Liu, D.; Wu, Y.; Yuan, M. L.; Li, L.; Yang, J. Y. Effect of PLA/PCL/cinnamaldehyde antimicrobial packaging on physicochemical and microbial quality of button mushroom (Agaricus bisporus). Postharvest Biol. Technol. 2015, 99, 73-79.

(30) Zhao, H.; Yuan, J.; Yang, Q.; Xie, Y.; Cao, W.; Wang, S. Cinnamaldehyde in a Novel Intravenous Submicrometer Emulsion: Pharmacokinetics, Tissue Distribution, Antitumor Efficacy, and Toxicity. J. Agric. Food Chem. 2015, 63, 6386-6392.

(31) Liu, F.; Saricaoglu, F. T.; Avena-Bustillos, R. J.; Bridges, D. F.; Takeoka, G. R.; Wu, V. C. H.; Chiou, B. S.; Wood, D. F.; McHugh, T. H.; Zhong, F. Preparation of Fish Skin Gelatin-Based Nanofibers Incorporating Cinnamaldehyde by Solution Blow Spinning. Int. J. Mol. Sci. 2018, 19, 618.

(32) Rieger, K. A.; Schiffman, J. D. Electrospinning an essential oil: cinnamaldehyde enhances the antimicrobial efficacy of chitosan/ poly(ethylene oxide) nanofibers. Carbohydr. Polym. 2014, 113, 561568.

(33) Maftoonazad, N.; Shahamirian, M.; John, D.; Ramaswamy, H. Development and evaluation of antibacterial electrospun pea protein isolate-polyvinyl alcohol nanocomposite mats incorporated with cinnamaldehyde. Mater. Sci. Eng., C 2019, 94, 393-402.

(34) Chun, J.-Y.; Jo, Y.-J.; Bjrapha, P.; Choi, M.-J.; Min, S.-G. Antimicrobial Effect of alpha- or beta-Cyclodextrin Complexes with Trans-Cinnamaldehyde Against Staphylococcus aureus and Escherichia coli. Drying Technol. 2015, 33, 377-383.

(35) Liu, Y.; Liang, X.; Zhang, R.; Lan, W.; Qin, W. Fabrication of Electrospun Polylactic Acid/Cinnamaldehyde/ $\beta$-Cyclodextrin Fibers as an Antimicrobial Wound Dressing. Polymers 2017, 9, 464.
(36) Carlotti, M. E.; Sapino, S.; Cavalli, R.; Trotta, M.; Trotta, F.; Martina, K. Inclusion of cinnamaldehyde in modified gammacyclodextrins. J. Incl. Phenom. Macrocycl. 2007, 57, 445-450.

(37) Davaatseren, M.; Jo, Y.-J.; Hong, G.-P.; Hur, H. J.; Park, S.; Choi, M.-J. Studies on the Anti-Oxidative Function of transCinnamaldehyde-Included -Cyclodextrin Complex. Molecules 2017, 22, 1868.

(38) Chen, H.; Li, L.; Ma, Y.; Mcdonald, T. P.; Wang, Y. Development of active packaging film containing bioactive components encapsulated in beta-cyclodextrin and its application. Food Hydrocolloids 2019, 90, 360-366.

(39) Sun, Q.; Tang, P.; Zhao, L.; Pu, H.; Zhai, Y.; Li, H. Mechanism and structure studies of cinnamaldehyde/cyclodextrins inclusions by computer simulation and NMR technology. Carbohydr. Polym. 2018, 194, 294-302.

(40) Wen, P.; Zhu, D.-H.; Wu, H.; Zong, M.-H.; Jing, Y.-R.; Han, S.Y. Encapsulation of cinnamon essential oil in electrospun nanofibrous film for active food packaging. Food Control 2016, 59, 366-376.

(41) Wen, P.; Zhu, D.-H.; Feng, K.; Liu, F.-J.; Lou, W.-Y.; Li, N.; Zong, M.-H.; Wu, H. Fabrication of electrospun polylactic acid nanofilm incorporating cinnamon essential oil/beta-cyclodextrin inclusion complex for antimicrobial packaging. Food Chem. 2016, 196, 996-1004.

(42) Higuchi, T.; Connors, K. A. Phase solubility techniques. Adv. Anal. Chem. Instrum. 1965, 4, 117-212.

(43) Kohn, W.; Sham, L. J. Self-Consistent Equations Including Exchange and Correlation Effects. Phys. Rev. 1965, 140, A1133A1138.

(44) Hohenberg, P.; Kohn, W. Inhomogeneous Electron Gas. Phys. Rev. 1964, 136, B864-B871.

(45) Kresse, G.; Furthmuller, J. Efficient iterative schemes for $a b$ initio total-energy calculations using a plane-wave basis set. Phys. Rev. B: Condens. Matter Mater. Phys. 1996, 54, 11169-11186.

(46) Kresse, G.; Furthmuller, J. Efficiency of ab-initio total energy calculations for metals and semiconductors using a plane-wave basis set. Comput. Mater. Sci. 1996, 6, 15-50.

(47) Perdew, J. P.; Burke, K.; Ernzerhof, M. Generalized Gradient Approximation Made Simple. Phys. Rev. Lett. 1996, 77, 3865-3868.

(48) Grimme, S. Semiempirical GGA-type density functional constructed with a long-range dispersion correction. J. Comput. Chem. 2006, 27, 1787-1799.

(49) Blöchl, P. E. Projector augmented-wave method. Phys. Rev. B: Condens. Matter Mater. Phys. 1994, 50, 17953-17979.

(50) Mathew, K.; Sundararaman, R.; Letchworth-Weaver, K.; Arias, T. A.; Hennig, R. G. Implicit solvation model for density-functional study of nanocrystal surfaces and reaction pathways. J. Chem. Phys. 2014, 140, 084106

(51) Li, X.; Kanjwal, M. A.; Lin, L.; Chronakis, I. S. Electrospun polyvinyl-alcohol nanofibers as oral fast-dissolving delivery system of caffeine and riboflavin. Colloids Surf., B 2013, 103, 182-188.

(52) Cho, E.; Jung, S. Supramolecular Complexation of Carbohydrates for the Bioavailability Enhancement of Poorly Soluble Drugs. Molecules 2015, 20, 19620-19646.

(53) Uyar, T.; Besenbacher, F. Electrospinning of uniform polystyrene fibers: The effect of solvent conductivity. Polymer 2008, 49, 5336-5343.

(54) Kayaci, F.; Uyar, T. Solid inclusion complexes of vanillin with cyclodextrins: their formation, characterization, and high-temperature stability. J. Agric. Food Chem. 2011, 59, 11772-11778.

(55) Yuan, W.; Teo, C. H. M.; Yuk, H.-G. Combined antibacterial activities of essential oil compounds against Escherichia coli O157:H7 and their application potential on fresh-cut lettuce. Food Control 2019, 96, 112-118.

(56) Tu, X.-F.; Hu, F.; Thakur, K.; Li, X.-L.; Zhang, Y.-S.; Wei, Z.-J. Comparison of antibacterial effects and fumigant toxicity of essential oils extracted from different plants. Ind. Crops Prod. 2018, 124, 192200.

(57) Valero, M.; Giner, M. J. Effects of antimicrobial components of essential oils on growth of Bacillus cereus INRA L2104 in and the 
sensory qualities of carrot broth. Int. J. Food Microbiol. 2006, 106, 9094.

(58) Pei, R. S.; Zhou, F.; Ji, B.-P.; Xu, J. Evaluation of Combined Antibacterial Effects of Eugenol, Cinnamaldehyde, Thymol, and Carvacrol against E-coli with an Improved Method. J. Food Sci. 2009, 74, M379-M383.

(59) Sanla-Ead, N.; Jangchud, A.; Chonhenchob, V.; Suppakul, P. Antimicrobial Activity of Cinnamaldehyde and Eugenol and Their Activity after Incorporation into Cellulose-based Packaging Films. Packag. Technol. Sci. 2012, 25, 7-17.

(60) Uzunlu, S.; Niranjan, K. Laboratory antimicrobial activity of cinnamaldehyde and pomegranate-based polycaprolactone films. J. Appl. Polym. Sci. 2017, 134, 45347.

(61) Liang, H.; Yuan, Q. P.; Vriesekoop, F.; Lv, F. Effects of cyclodextrins on the antimicrobial activity of plant-derived essential oil compounds. Food Chem. 2012, 135, 1020-1027.

(62) Yalkowsky, S. H.; He, Y.; Jain, P. Handbook of Aqueous Solubility Data, 2nd ed.; CRC Press: Boca Raton, FL, 2010; p xii, 1608. 